\title{
Pattern formation in martensitic thin films
}

\author{
Y. C. Shu ${ }^{\text {a) }}$ and J. H. Yen \\ Institute of Applied Mechanics, National Taiwan University, Taipei 106, Taiwan, Republic of China
}

(Received 4 June 2007; accepted 16 June 2007; published online 10 July 2007)

\begin{abstract}
Pattern formation in martensitic materials refers to the accommodation problem of how to mix martensitic variants coherently to minimize the strain energy. A framework motivated by energy-minimizing multirank laminated patterns is proposed to study this problem in martensitic films. It is found that the interfaces between the variants of martensite can be quite different in thin films than in bulk materials, and they typically have a simpler structure. Various intriguing and fascinating self-accommodation patterns are predicted for martensitic thin films with different orientations. The results are in good agreement with the Bhattacharya-James thin-film theory [K. Bhattacharya and R. D. James, J. Mech. Phys. Solids 47, 531 (1999)] as well as with experimental observations. (C) 2007 American Institute of Physics. [DOI: 10.1063/1.2756320]
\end{abstract}

Martensitic materials undergo a first-order diffusionless phase transformation during which there is a sudden change in the crystal structure at a certain temperature. ${ }^{1}$ A number of recent investigations have suggested that the characteristic distortions of martensite can be exploited to create tiny machines. ${ }^{2}$ The key, then, to achieve the exceptional potential of these materials is to design devices that can take full advantage of the inherent martensitic microstructure. However, while much work has addressed the problem of pattern formation in martensitic bulk crystals, little consideration had given to this phenomenon in martensitic thin films until recently. There are various reasons for that. In particular, the anisotropic and highly nonlinear properties of martensitic materials have made it difficult to develop suitable theories to describe their behavior in slender structures. Bhattacharya and James $^{3}$ have employed the notion of $\Gamma$-convergence to derive a theory of martensitic single crystal films, and $\mathrm{Shu}^{4}$ has extended it to polycrystal films. They have shown that the coherence requirement is weakened in thin films, and this enables a novel strategy for the design of microactuators. 5,6 Such a strategy, on the other hand, requires a detailed understanding of microstructure and its evolution under stress in martensitic films. This in turn calls for an appropriate model that can capture the spirit of the Bhattacharya-James theory while also serving as a convenient tool to evaluate various conditions in design.

The key feature of a martensitic phase transformation is the microstructure it generates. The high-temperature austenite phase is cubic, while the low-temperature martensite phase has less symmetry. This gives rise to symmetry-related variants, which are identical crystal lattices of martensite with different orientations. The transformation from the austenite to the $i$ th variant of martensite is described by the transformation strain $\varepsilon^{(i)}$, and $i=1, \ldots, N$ where $N$ is the number of martensitic variants. It can be determined from the change of symmetry and lattice parameters. In the case of cubic to trigonal transformation, $N=4$ and

\footnotetext{
a) Author to whom correspondence should be addressed; electronic address: yichung@spring.iam.ntu.edu.tw
}

$$
\begin{aligned}
& \varepsilon^{(1)}=\left(\begin{array}{ccc}
\alpha & \delta & \delta \\
\delta & \alpha & \delta \\
\delta & \delta & \alpha
\end{array}\right), \quad \varepsilon^{(3)}=\left(\begin{array}{ccc}
\alpha & -\delta & \delta \\
-\delta & \alpha & -\delta \\
\delta & -\delta & \alpha
\end{array}\right), \\
& \varepsilon^{(2)}=\left(\begin{array}{ccc}
\alpha & -\delta & -\delta \\
-\delta & \alpha & \delta \\
-\delta & \delta & \alpha
\end{array}\right), \quad \varepsilon^{(4)}=\left(\begin{array}{ccc}
\alpha & \delta & -\delta \\
\delta & \alpha & -\delta \\
-\delta & -\delta & \alpha
\end{array}\right),
\end{aligned}
$$

where $\alpha$ and $\delta$ are material parameters. Above, the crystal and reference bases are assumed to coincide. Let $\varepsilon^{*}$ be a macroscopically homogeneous strain. It is recoverable if it can be obtained by a coherent mixture of martensitic variants. Indeed, any recoverable strain in this case can be achieved by a rank-3 laminate of variants; ${ }^{7}$ i.e.,

$$
\varepsilon^{*}=\sum_{i=1}^{4} \gamma_{i} \varepsilon^{(i)},
$$

where $\gamma_{i}$ is the global volume fraction of the $i$ th variant and can be expressed in terms of

$$
\begin{aligned}
& \gamma_{1}=\mu_{1}, \\
& \gamma_{2}=\left(1-\mu_{1}\right) \mu_{2}, \\
& \gamma_{3}=\left(1-\mu_{1}\right)\left(1-\mu_{2}\right) \mu_{3}, \\
& \gamma_{4}=\left(1-\mu_{1}\right)\left(1-\mu_{2}\right)\left(1-\mu_{3}\right),
\end{aligned}
$$

and $\mu_{j}$ is the local volume fraction of some combinations of martensitic variants in the $j$ th rank of laminate $(j=1, \ldots, N$ $-1)$. Notice that $\sum_{i=1}^{N} \gamma_{i}=1$. This idea of using an energyminimizing multirank laminated microstructure has recently been applied to the study of domain switching in ferroelectric crystals under combined electromechanical loadings (see Fig. 1 therein $^{8}$ ).

Instead, let $\varepsilon^{*}$ here be interpreted as a locally inhomogeneous strain; i.e, $\varepsilon^{*}(\mathbf{x})$ is equal to one of $\varepsilon^{(i)}$ at each point $\mathbf{x}$. The above multirank laminated structure suggests that this is feasible if each $\mu_{j}(\mathbf{x})$ is equal to 0 or 1 at each point $\mathbf{x}$. Thus, when $\gamma_{k}(\mathbf{x})=1$ at $\mathbf{x}, \gamma_{l}(\mathbf{x})=0$ if $l \neq k$. We now use this idea to study the morphology of martensitic microstructure. 


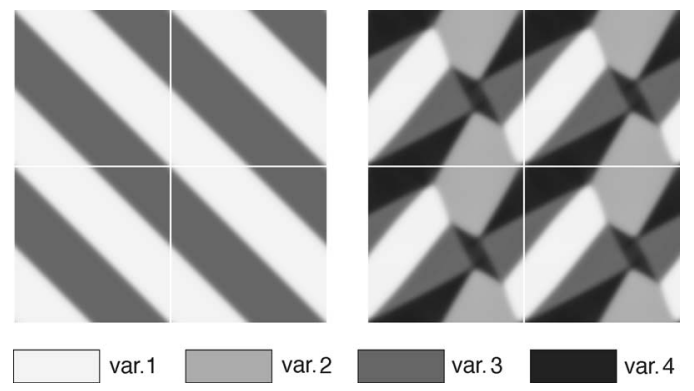

FIG. 1. LHS and RHS are self-accommodation patterns in (001) and (111) films. As periodic conditions are taken in the simulations, four identical patterns are packed together to obtain a better image.

Let $\mu_{j}(\mathbf{x})$ be relaxed to continuously vary across the sharp interfaces at the boundaries of martensitic variants. The free energy of a martensite at some fixed temperature below the critical temperature is described in terms of the field variables $\mu_{j}$ by

$$
\begin{aligned}
& \mathcal{I}(\boldsymbol{\mu})=\int_{\Omega}\left\{W^{\text {int }}(\boldsymbol{\mu})+W^{a}(\boldsymbol{\mu})+W^{\text {elas }}(\boldsymbol{\mu})\right\} d \mathbf{x}, \\
& W^{\text {int }}(\boldsymbol{\mu})=A|\nabla \boldsymbol{\mu}|^{2}, \\
& W^{a}(\boldsymbol{\mu})=K \sum_{j=1}^{N-1} \mu_{j}^{2}\left(1-\mu_{j}\right)^{2}, \\
& W^{\text {elas }}(\boldsymbol{\mu})=\frac{1}{2}\left[\varepsilon-\varepsilon^{*}(\boldsymbol{\mu})\right] \cdot \mathbf{C}\left[\varepsilon-\varepsilon^{*}(\boldsymbol{\mu})\right],
\end{aligned}
$$

subject to the constraint,

$$
\begin{aligned}
& \nabla \cdot \boldsymbol{\sigma}=0, \\
& \boldsymbol{\sigma}=\mathbf{C}\left[\varepsilon-\varepsilon^{*}(\boldsymbol{\mu})\right],
\end{aligned}
$$

where $\boldsymbol{\mu}=\left(\mu_{1}, \mu_{2}, \ldots, \mu_{N-1}\right), \varepsilon$ is the compatible linear strain, and $\mathbf{C}$ is the elastic modulus and is approximated to be the same for all phases.

Each of the terms in Eq. (4) has a physical interpretation. The first term with $A>0$, called the interfacial energy density, penalizes changes in the field variables and thus is interpreted as the energy of forming a martensitic interface. The second and third terms, with $K>0$, are the anisotropy and elastic energy densities. The sum of these two denotes the energetic cost that the crystal must pay if the field variables and strain deviate from the preferred states; thus, this builds in the information that the crystal prefers a certain spontaneous strain.

We postulate that the martensitic microstructure is obtained by minimizing the total free energy in Eq. (4) under the constraint in Eq. (5). However, it is not an easy task. Alternatively, the energy is decreasing if it follows the path ${ }^{9}$

$$
\frac{\partial \boldsymbol{\mu}}{\partial t}=-L \frac{\delta \mathcal{I}}{\delta \boldsymbol{\mu}}=L \mathbf{F},
$$

where $L>0$ is the mobility and $\mathbf{F}=-\delta \mathcal{I} / \delta \boldsymbol{\mu}$ is the thermodynamic driving force defined by the variational derivative of the free energy. It is equal to $\mathbf{F}^{\text {int }}+\mathbf{F}^{a}+\mathbf{F}^{\text {elas }}$, where $\mathbf{F}^{\text {int }}$ $=2 A \nabla^{2} \boldsymbol{\mu}$ is the driving force for the coarsening of microstructure, $\mathbf{F}^{a}=-\partial W^{a}(\boldsymbol{\mu}) / \partial \boldsymbol{\mu}$ is the driving force for setting $\boldsymbol{\mu}$
Downloaded 21 Dec 2008 to 140.112 .113 .225 . Redistribution subject to AIP license or copyright; see http://apl.aip.org/apl/copyright.jsp
TABLE I. Compatible interfacial normals in (110) films.

\begin{tabular}{cccc}
\hline \hline Variants & 1,2 or 1,3 & 1,4 & 2,4 or 3,4 \\
\hline$\{100\}$ type & $(1,0)$ & $(0,1)$ & $(1,0)$ \\
$\{110\}$ type & $(1, \sqrt{2})$ & $(1,0)$ & $(-1, \sqrt{2})$ \\
\hline \hline
\end{tabular}

as much close to the lowest points in the energy wells as possible, and $\mathbf{F}^{\text {elas }}=\mathbf{C}\left[\varepsilon-\varepsilon^{*}(\boldsymbol{\mu})\right] \cdot \partial \varepsilon^{*}(\boldsymbol{\mu}) / \partial \boldsymbol{\mu}$ is the driving force for the refinement of microstructure to accommodate the boundary constraints.

The present method is different from the conventional phase field models developed by Jin et al., ${ }^{10}$ Lookman et al.,${ }^{11}$ Salje et al., ${ }^{12}$ Slutsker et al. ${ }^{13}$ and Li et al., ${ }^{14,15}$ for martensitic and ferroelectric microstructure evolutions. Their approaches choose a suitable set of order parameters and the special polynomial expansions of them at high orders for a particular transformation. Instead, we choose a set of field variables motivated by the hierarchical structure of multirank laminates as in Eq. (3). Besides, the anisotropy energy density $W^{a}(\boldsymbol{\mu})$ in Eq. (4) is explicitly given irrespective of different types of martensitic transformation under consideration.

We now apply Eq. (6) to the study of pattern formation in martensitic thin films undergoing cubic to trigonal transformation at a fixed temperature below the critical one. Therefore, the influence of the austenite variant is insignificant here. We consider a martensitic single crystal film released from the substrate but constrained on its lateral boundaries. Suppose the thickness of the film is much smaller than the lateral extent. In this situation, Bhattacharya and James ${ }^{3}$ have shown that the out-of-plane strain incompatibility can be neglected. Therefore, we need to consider only the in-plane components of the transformation strains $\varepsilon^{(i)}$ in Eq. (1). Let $\mathbf{R}_{(001)}, \mathbf{R}_{(110)}$, and $\mathbf{R}_{(111)}$ be the proper

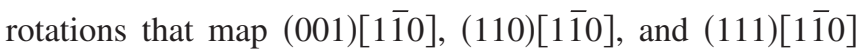
back to the identity. Therefore, the transformation strains in the reference basis become $\mathbf{R}_{(001)} \varepsilon^{(i)} \mathbf{R}_{(001)}^{T}, \mathbf{R}_{(110)} \varepsilon^{(i)} \mathbf{R}_{(110)}^{T}$, and $\mathbf{R}_{(111)} \varepsilon^{(i)} \mathbf{R}_{(111)}^{T}$ for $i=1, \ldots, 4$. The matrices containing the in-plane components of the above transformation strains are denoted by $\bar{\varepsilon}_{(001)}^{(i)}, \bar{\varepsilon}_{(110)}^{(i)}$, and $\bar{\varepsilon}_{(111)}^{(i)}$.

We use Ti-Ni in the $R$ phase as the representative material. This gives $\alpha=0$ and $\delta=0.0047$ in Eq. (1). ${ }^{16}$ The elastic moduli of Ti-Ni single crystals are not available, therefore, we take $C_{11}=C_{22}=80 \mathrm{GPa}, C_{12}=20 \mathrm{GPa}, C_{66}=30 \mathrm{GPa}$, and $C_{16}=C_{26}=0$ (Voigt notation), which are typical parameters for Ti-Ni polycrystals. The present formulation involves only two parameters. The first one $A$ is related to the length
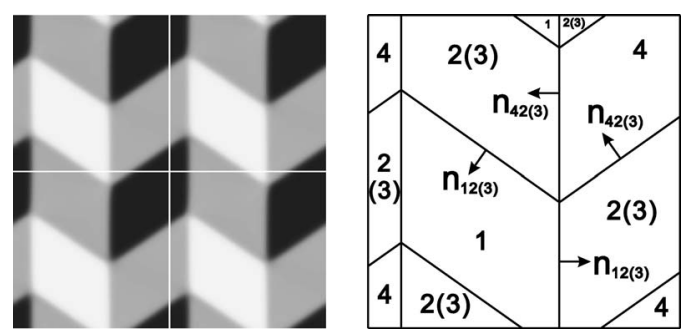

FIG. 2. Self-accommodation pattern in (110) films. icense or copyright; see http://apl.aip.org/apl/copyright.jsp 

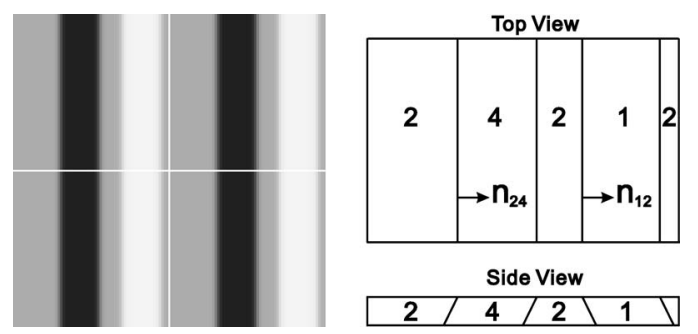

FIG. 3. Another possible self-accommodation pattern in (110) films.

scale of the microstructure and can be grouped to a dimensionless parameter $D=(A / K) / l_{0}^{2}$, where $l_{0}$ is the size of the simulation. We take $D=0.0001{ }^{17}$ The other parameter $K$ is chosen such that the energy densities $W^{a}$ and $W^{\text {elas }}$ are of the same order. The periodic boundary conditions are taken for simulations, and the fast Fourier transform is employed to enhance the speed of computation. As the nucleation problem is not considered in the present study, ${ }^{10,18}$ we take the random initial conditions. ${ }^{19}$

For (001) films, variants 1 and 4 and variants 2 and 3 are indistinguishable, since $\bar{\varepsilon}_{(001)}^{(1)}=\bar{\varepsilon}_{(001)}^{(4)}$ and $\bar{\varepsilon}_{(001)}^{(2)}=\bar{\varepsilon}_{(001)}^{(3)}$. Therefore, there are only two distinct variants, and the selfaccommodation pattern is the lamellar type, as shown in the left-hand side (LHS) of Fig. 1, where different variants are presented by different gray levels. While such a lamellar pattern is not a basic unit for self-accommodation in bulk trigonal martensites, ${ }^{20}$ it holds true in thin films since the in-plane average of transformation strains is zero. As a result, this pattern is commonly observed in many (001) trigonal films. ${ }^{21}$

For (110) films, variants 2 and 3 are indistinguishable, since $\bar{\varepsilon}_{(110)}^{(2)}=\bar{\varepsilon}_{(110)}^{(3)}$. Therefore, there are three distinct variants. In a bulk trigonal martensite, there are two typical interfaces: one is $\{100\}$ type and the other is $\{110\}$ type. The corresponding interfacial normals predicted based on the Bhattacharya-James thin-film theory are listed in Table I. The simulation results give two distinct patterns. The first one, in Fig. 2, is similar to the commonly observed "herring-bone" pattern in trigonal martensites. ${ }^{20}$ Another simpler pattern is shown in the LHS of Fig. 3, which is not an allowable pattern in bulk martensites, since the third components in the interfacial normals are different, as can be seen in the righthand side (RHS) of Fig. 3. However, it is a legitimate one in thin films, and this confirms that martensitic materials can
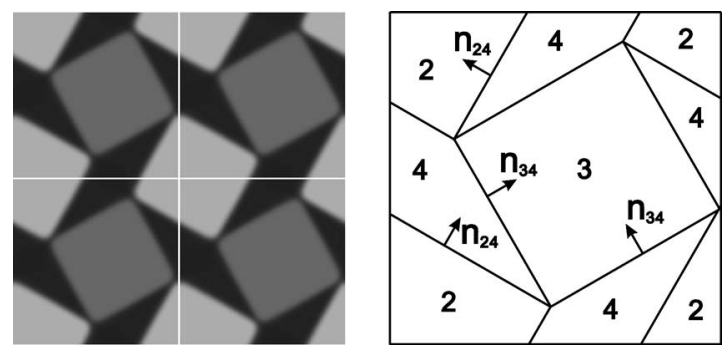

FIG. 4. Pattern in (111) films under biaxial tension.
TABLE II. Compatible interfacial normals in (111) films.

\begin{tabular}{ccccccc}
\hline \hline Variants & 1,2 & 1,3 & 1,4 & 2,3 & 2,4 & 3,4 \\
\hline$\{100\}$ type & $(\sqrt{3}, 1)$ & $(-\sqrt{3}, 1)$ & $(0,1)$ & $(0,1)$ & $(-\sqrt{3}, 1)$ & $(\sqrt{3}, 1)$ \\
$\{110\}$ type & $(\sqrt{3}, 1)$ & $(-\sqrt{3}, 1)$ & $(0,1)$ & $(1,0)$ & $(1, \sqrt{3})$ & $(1,-\sqrt{3})$ \\
\hline \hline
\end{tabular}

form many more interfaces in a thin film than in bulk. In addition, the interfacial normals in these two simulations agree very well with those listed in Table I.

For (111) films, all of the in-plane transformation strains are different in this case, and a self-accommodation pattern containing all of these four martensitic variants is shown in the RHS of Fig. 1. Next, to see how the microstructure evolves under stress, consider a biaxial tension $\boldsymbol{\sigma}^{*}=\sigma_{0} \mathbf{I}$ applied in the plane of the film, where $\sigma_{0}>0$ and $\mathbf{I}$ is the identity matrix. This case is important since these films are typically stressed under pressure when used in micropumps. Figure 4 shows the coalescence of martensitic variants under stress. Variant 1 disappears and the volume fractions of phases 2, 3, and 4 are identical. This can be explained by an energetic argument: $-\boldsymbol{\sigma}^{*} \cdot \bar{\varepsilon}_{(111)}^{(2)}=-\boldsymbol{\sigma}^{*} \cdot \bar{\varepsilon}_{(111)}^{(3)}=-\boldsymbol{\sigma}^{*} \cdot \bar{\varepsilon}_{(111)}^{(4)}<0$ while $-\boldsymbol{\sigma}^{*} \cdot \bar{\varepsilon}_{(111)}^{(1)}>0$. Finally, according to Table II, the resulting morphology evolves along a compatible path to reduce the strain energy.

The authors are glad to acknowledge the financial support of the National Science Council of Taiwan under Grant No. 95-2221-E-002-166.

${ }^{1}$ S. K. Wu and H. C. Lin, Mater. Chem. Phys. 64, 81 (2000).

${ }^{2}$ K. Bhattacharya and R. D. James, Science 307, 53 (2005).

${ }^{3}$ K. Bhattacharya and R. D. James, J. Mech. Phys. Solids 47, 531 (1999).

${ }^{4}$ Y. C. Shu, Arch. Ration. Mech. Anal. 153, 39 (2000).

${ }^{5}$ K. Bhattacharya, A. DeSimone, K. F. Hane, R. D. James, and C. J. Palmstrøm, Mater. Sci. Eng., A 273-275, 685 (1999).

${ }^{6}$ Y. C. Shu, Mater. Trans. 43, 1037 (2002).

${ }^{7}$ K. Bhattacharya, Continuum Mech. Thermodyn. 5, 205 (1993).

${ }^{8}$ Y. C. Shu, J. H. Yen, J. Shieh, and J. H. Yeh, Appl. Phys. Lett. 90, 172902 (2007).

${ }^{9}$ Y. C. Shu, M. P. Lin, and K. C. Wu, Mech. Mater. 36, 975 (2004).

${ }^{10}$ Y. M. Jin, A. Artemev, and A. G. Khachaturyan, Acta Mater. 49, 2309 (2001).

${ }^{11}$ T. Lookman, S. R. Shenoy, K. Ø. Rasmussen, A. Saxena, and A. R. Bishop, Phys. Rev. B 67, 024114 (2003).

${ }^{12}$ E. K. H. Salje, H. Zhang, D. Schryvers, and B. Bartova, Appl. Phys. Lett. 90, 221903 (2007).

${ }^{13}$ J. Slutsker, A. Artemev, and A. L. Roytburd, J. Appl. Phys. 91, 9049 (2002).

${ }^{14}$ Y. L. Li, S. Y. Hu, Z. K. Liu, and L. Q. Chen, Appl. Phys. Lett. 78, 3878 (2001).

${ }^{15}$ Y. L. Li, S. Y. Hu, Z. K. Liu, and L. Q. Chen, Appl. Phys. Lett. 81, 427 (2002).

${ }^{16}$ S. Miyazaki, S. Kimura, and K. Otsuka, Philos. Mag. A 57, 467 (1988).

${ }^{17}$ A. Artemev, Y. Jin, and A. G. Khachaturyan, Acta Mater. 49, 1165 (2001).

${ }^{18}$ K. Dayal and K. Bhattacharya, Acta Mater. 55, 1907 (2007).

${ }^{19}$ K. Ø. Rasmussen, T. Lookman, A. Saxena, A. R. Bishop, R. C. Albers, and S. R. Shenoy, Phys. Rev. Lett. 87, 055704 (2001).

${ }^{20}$ T. Fukuda, T. Saburi, K. Doi, and S. Nenno, Mater. Trans., JIM 33, 271 (1992).

${ }^{21}$ S. K. Streiffer, C. B. Parker, A. E. Romanov, M. J. Lefevre, L. Zhao, J. S. Speck, W. Pompe, C. M. Foster, and G. R. Bai, J. Appl. Phys. 83, 2742 (1998). 